\title{
Artifact of the phonon-induced localization by variational calculations in the spin-boson model
}

\author{
Zhi-De Chen* and Hang Wong \\ Department of Physics, Jinan University, Guangzhou 510632, China
}

\begin{abstract}
We present energy and free energy analyses on all variational schemes used in the spin-boson model at both $T=0$ and $T \neq 0$. It is found that all the variational schemes have fail points, at where the variational schemes fail to provide a lower energy (or a lower free energy at $T \neq 0$ ) than the displaced-oscillator ground state and therefore the variational ground state becomes unstable, which results in a transition from a variational ground state to a displaced oscillator ground state when the fail point is reached. Such transitions are always misidentified as crossover from a delocalized to localized phases in variational calculations, leading to an artifact of phonon-induced localization. Physics origin of the fail points and explanations for different transition behaviors with different spectral functions are found by studying the fail points of the variational schemes in the single mode case.

PACS numbers: 03.65.Yz, 03.65.Xp, 73.40.Gk
\end{abstract}

\section{INTRODUCTION}

Variational method is one of the important tools in tracing the ground state of many body systems. $\stackrel{1}{*}$ In dealing with systems having interaction with phonons, the variational method based on displaced-oscillator state (DOS) plays an important role. Some well-known examples in condensed matter physics are polaronphonon, exciton-phonon systems, 2,3,4 and spin-boson model, which is an important toy model in dissipative quantum systems, $, 4,6$ In fact, the coupling to a phonon bath in all the mentioned systems has the same bilinear coupling form. One important issue in the mentioned systems is the so-called phonon-induced localization. In exciton-phonon (or polaron-phonon) systems, the phonon-induced localization is called the selftrapped transition, while in the spin-boson model, it is stated as the crossover from a delocalized to localized phases $, 5,6,8$ Such a crossover is now considered as some kind of quantum phase transition called boundary phase transition. 9,10 In general, variational calculation has been serving as an important tool in dealing with the phononinduced localization, however, the predicted transitions in exciton-phonon (or polaron-phonon) systems turned out to be false ones but the answer to why the variational calculation fails was not found. 11 Variational calculation on the crossover problem in the spin-boson model has a long history and the first variational scheme is based on the DOS $, 12,13,14$ It was shown that, in strong coupling regime, the coupling to phonon bath can lead to both displacement and deformation of the oscillator and the so-called displaced-squeezed-state (DSS) is energy favorite, leading to a variational calculation based on the DSS. 15,16 Later, a hybrid variational scheme based on the DSS was also developed. ${ }^{17}$ At finite temperature regime, the variational calculation based on the DOS was done for both the Ohmic and sub-Ohmic cases and a discontinuous crossover was found, $13,18,19,20$ and recently, it is shown that the variational calculation based on the DSS fails in thermodynamical limit 21

Despite of its long history, the result from variational calculations is far from satisfactory. The basic problem is that the predicted crossover point is variationalscheme dependent. For example, in the Ohmic case, the crossover point by the variational calculation based on the DOS was $\alpha_{c}=1,12$ while that based on the DSS gave $\alpha_{c}=2,15,16$ and the hybrid variational scheme predicted a $\alpha_{c}$ lay between.17 The transition behavior predicted by the variational calculations is also in controversy. The variational calculations based on both the DOS and DSS predicted a continuous transition in the Ohmic case but discontinuous transition behavior was also found in the hybrid variational scheme 17 In the sub-Ohmic case, all the variational calculations predicted a discontinuous transition, ${ }^{18,19}$ but non-perturbative numerical calculations, like numerical renormalization group (NRG) and density-matrix renormalization group (DMRG), found a continuous transition instead ${ }^{23,24,25}$ The situation in the case of $T \neq 0$ is also confusing. Variational calculations predicted a discontinuous transition at $T \neq 0$ even in the super-Ohmic case,$\stackrel{22}{2}$ a result which is in confliction with the known conclusion that the crossover only happens in the Ohmic and sub-Ohmic cases ${ }^{5,6,7,8}$ All these discrepancies make the crossover predicted by the variational calculations in question, e.g., is it an artifact as in the case of polaron-phonon systems? Clearing up this issue is important for understanding the result by variational calculations and further application of variational methods to other systems. This is the motivation of the present paper.

By employing energy analysis on the variational schemes used in the spin-boson model, we shown that the predicted crossover is just an artifact of the variational schemes. We will show that all the variational schemes mentioned above have fail points, at where variational schemes fail to provide a lower energy than that of the displaced-oscillator ground state, leading to a transition from the variational ground state to the displacedoscillator ground state when the fail point is reached. 
In variational calculations, such a transition is always misidentified as a crossover from a delocalized to localized phases and the fail point is misinterpreted as a crossover point. The result we found can help to resolve the controversy mentioned above.

The rest of the paper is organized as follows: The fail points of the variational schemes at both $T=0$ and $T \neq 0$ are demonstrated in the next section. In Sec III, physical origin of the fail point and different transition behaviors in both the Ohmic and sub-Ohmic cases are analyzed by studying the variational schemes in the single mode case. Conclusion and discussion are presented in the last section.

\section{FAIL POINTS OF THE VARIATIONAL SCHEMES USED IN THE SPIN-BOSON MODEL}

The Hamiltonian of the spin-boson model is given by (setting $\hbar=1)^{5,6}$

$$
\hat{H}=-\frac{\Delta_{0}}{2} \hat{\sigma}_{x}+\sum_{k} \hat{b}_{k}^{\dagger} \hat{b}_{k} \omega_{k}+\hat{\sigma}_{z} \sum_{k} c_{k}\left(\hat{b}_{k}^{\dagger}+\hat{b}_{k}\right),
$$

where $\hat{\sigma}_{i}(i=x, y, z)$ is the Pauli matrix, $\hat{b}_{k}\left(\hat{b}_{k}^{\dagger}\right)$ is the annihilation (creation) operator of the $k$ th phonon mode with energy $\omega_{k}$ and $c_{k}$ is the corresponding coupling parameter. As is known, the solution of this model depends on the so-called bath spectral function that is defined as $J(\omega)=\pi \sum_{k} c_{k}^{2} \delta\left(\omega-\omega_{k}\right), \underline{5,6}$ Usually the spectral function has a power law form, i.e.,

$$
J(\omega)=\frac{\pi}{2} \alpha \omega^{s} \omega_{D}^{1-s}, \quad 0<\omega<\omega_{D},
$$

where $\omega_{D}$ is the cut-off frequency and $\alpha$ is a dimensionless coupling strength which characterizes the dissipation strength, 6 The property of the bath is characterized by the parameter $s$, i.e., $0<s<1, s=1$, and $s>1$ represent respectively the sub-Ohmic, Ohmic, and superOhmic cases.

It is well known that, with the bilinear coupling form given in the last term of Eq.(1), the Hamiltonian can be diagonalized by the so-called displaced-oscillator transformation in the case of $\Delta_{0}=0$. The ground state in this case is the displaced-oscillator ground state ${ }^{5,6,26}$

$$
\left|\Phi_{0}\right\rangle=\frac{1}{\sqrt{2}}\left(\left|\phi_{+}\right\rangle|\uparrow\rangle+\left|\phi_{-}\right\rangle|\downarrow\rangle\right),
$$

where $\left|\phi_{ \pm}\right\rangle=\exp \left\{\mp \sum_{k}\left(c_{k} / \omega_{k}\right)\left(\hat{b}_{k}-\hat{b}_{k}^{\dagger}\right)\right\}|0\rangle$, is the DOS, while $|\uparrow\rangle$ and $|\downarrow\rangle$ are the eigenstates of $\hat{\sigma}_{z}$. All the variational schemes used in the spin-boson model start from the DOS; analysis in details will be presented in the following.

\section{A. Fail points of the variational schemes at $T=0$}

As we have mentioned in the Sec I, there are three kinds of variational schemes used in the spin-boson model at $T=0$ and the key difference is the trial ground state. The trial ground state for the variational calculation based on the DOS is chosen as ${ }^{12}$

$$
\left|\Psi_{0}\right\rangle=\frac{1}{\sqrt{2}}\left(\left|\psi_{+}\right\rangle|\uparrow\rangle+\left|\psi_{-}\right\rangle|\downarrow\rangle\right)
$$

where

$$
\left|\psi_{ \pm}\right\rangle=\exp \left\{\mp \sum_{k} g_{k}\left(\hat{b}_{k}-\hat{b}_{k}^{\dagger}\right)\right\}|0\rangle
$$

and $g_{k}$ is the variational parameter. The trial ground state based on the DSS is given by ${ }^{15,16}$

$$
\left|\tilde{\Psi}_{0}\right\rangle=\frac{1}{\sqrt{2}}\left(\left|\varphi_{+}\right\rangle|\uparrow\rangle+\left|\varphi_{-}\right\rangle|\downarrow\rangle\right)
$$

where

$$
\left|\varphi_{ \pm}\right\rangle=\exp \left\{\mp \sum_{k} \frac{c_{k}}{\omega_{k}}\left(\hat{b}_{k}-\hat{b}_{k}^{\dagger}\right)\right\} \exp \left\{-\sum_{k} \gamma_{k}\left(\hat{b}_{k}^{2}-\hat{b}_{k}^{\dagger 2}\right)\right\}|0\rangle,
$$

is the DSS and $\gamma_{k}$ is the variational parameter. The third variational scheme is a hybrid scheme of the above two variational schemes and the trial ground state is 17

$$
\left|\tilde{\Phi}_{0}\right\rangle=\frac{1}{\sqrt{2}}\left(\left|\tilde{\varphi}_{+}\right\rangle|\uparrow\rangle+\left|\tilde{\varphi}_{-}\right\rangle|\downarrow\rangle\right)
$$

with

$\left|\tilde{\varphi}_{ \pm}\right\rangle=\exp \left\{\mp \sum_{k} g_{k}\left(\hat{b}_{k}-\hat{b}_{k}^{\dagger}\right)\right\} \exp \left\{-\sum_{k} \gamma_{k}\left(\hat{b}_{k}^{2}-\hat{b}_{k}^{\dagger 2}\right)\right\}|0\rangle$

here both $g_{k}$ and $\gamma_{k}$ are the variational parameters.

All the variational schemes have almost the same calculation process and the main routine is as follows. Firstly, one used the trial ground state $\left|\Phi_{t g s}\right\rangle$ to calculate the trial ground state energy $E_{t}=\left\langle\Phi_{\text {tgs }}|\hat{H}| \Phi_{\text {tgs }}\right\rangle$, then the following variational condition

$$
\delta E_{t} / \delta g_{k}=\delta E_{t} / \delta \gamma_{k}=0
$$

can help to determine the variational parameters. In what follows, the trial ground state with the variational parameters determined from the above variational condition is called the variational ground state $\left|\Psi_{v}\right\rangle$. Variation of the tunneling splitting of the variational ground state with the dissipative strength $\alpha$ can tell how the crossover happens. Since all three variational calculations give the same crossover behavior in both the Ohmic and subOhmic cases, here we shall use the variational calculation based on the DOS as an example to show the details.

For variational scheme based on the DOS, the variational condition (10) leads to

$$
g_{k}=\frac{c_{k}}{\omega_{k}+\Delta_{0} \exp \left\{-2 \sum_{k} g_{k}^{2}\right\}},
$$

from which the self-consistent equation of the dressing factor $K_{1}$ can be found $12,14,19$

$$
K_{1}=f\left(K_{1}\right) \equiv \exp \left\{-\frac{2}{\pi} \int_{0}^{\omega_{D}} \frac{J(\omega)}{\left(\omega+K_{1} \Delta_{0}\right)^{2}} d \omega\right\},
$$


here $K_{1}=\Delta / \Delta_{0}$ and $\Delta$ is the tunneling splitting in presence of dissipation. The trivial and non-zero solutions of Eq. (12) represent respectively the localized $(\Delta=0)$ and delocalized $(\Delta \neq 0)$ phases. Variational calculation determines the crossover by examining the evolution of solutions of Eq. (12) with the dissipation strength $\alpha$. It turned out that, in the Ohmic case, there is only one non-zero solution which decreases to zero continuously as $\alpha$ approaches to 1 in the limit of $\Delta_{0} / \omega_{D} \ll 1$, showing a continuous crossover happens at $\alpha_{c}=1$. The situation in the sub-Ohmic case is more complicated; there are two non-zero solutions $\left(K_{1}^{\prime}\right.$ and $\left.K_{1}^{\prime \prime}\right)$ which disappear abruptly when $\alpha$ reaches some critical value. It has been shown that the crossover in this case is discontinuous and the crossover point should be determined according to the Landau theory: ${ }^{18,19}$ The situation for other variational schemes is the same except for the values of the critical points. It should be noted that the discontinuous transition behavior in the Ohmic case found in the hybrid variational scheme $\frac{17}{}$ can also be found in other variational schemes and the key point is to increase the value of $\Delta_{0} / \omega_{D}$. We find that, for $\Delta_{0} / \omega_{D} \geq 0.5$, a discontinuous transition appears in the Ohmic case for the variational scheme based on the DOS. The change of transition behaviors is shown in Fig. 1. Explanation for this result will be presented in next section.

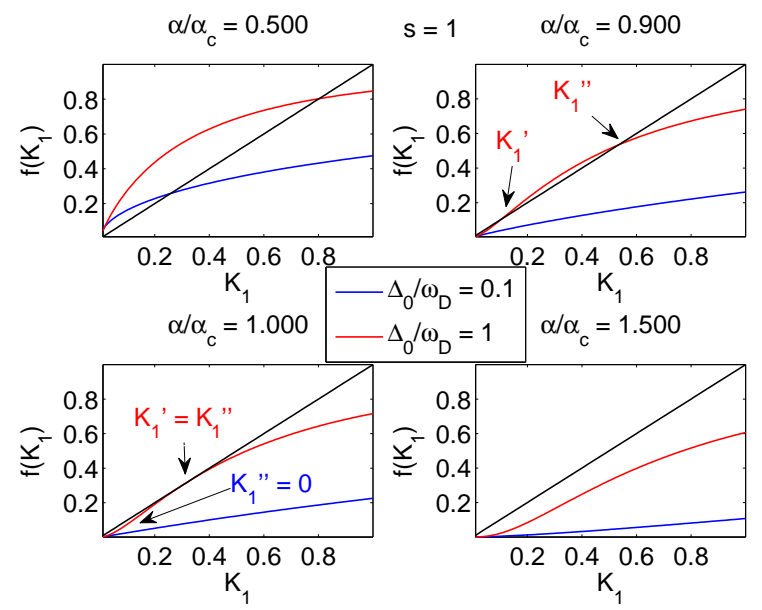

FIG. 1: (Color online) The evolution of solutions of the selfconsistent equation derived from the variational calculation based on the DOS in the Ohmic case. In the case of $\Delta_{0} / \omega_{D}=$ 0.1 , there is only one non-zero solution and the transition behavior is continuous. However, in the case of $\Delta_{0} / \omega_{D}=1$, there are two non-zero solutions $K_{1}^{\prime}$ and $K_{1}^{\prime \prime}$ and the transition behavior becomes discontinuous as $\alpha$ approaches $\alpha_{c}$.

Now we turn to show how variational schemes fail as $\alpha$ increases. As we have mentioned, the starting point of all the variational schemes is DOS. Consequently, for a successful variational scheme, the variational ground states $\left|\Psi_{v}\right\rangle$ should be a better approximation to the true ground state than the displaced-oscillator ground state $\left|\Phi_{0}\right\rangle$, which implies that $\left|\Psi_{v}\right\rangle$ should have a lower energy than $\left|\Phi_{0}\right\rangle$, e.g., the following condition

$$
E_{v}=\left\langle\Psi_{v}|\hat{H}| \Psi_{v}\right\rangle<E_{0}=\left\langle\Phi_{0}|\hat{H}| \Phi_{0}\right\rangle,
$$

should hold in a variational calculation, otherwise the variational scheme fails. Surprisingly, it is found that all the variational schemes fail when $\alpha$ increases to some critical value in both the Ohmic and sub-Ohmic cases. In the sub-Ohmic case, the variational ground state has a lower energy than the displaced-oscillator ground state when $\alpha \ll \alpha_{c}$. As $\alpha$ increases, both $E_{v}$ and $E_{0}$ decreases but $E_{0}$ decreases in a way faster than $E_{v}$, we have $E_{v}=$ $E_{0}$ when $\alpha=\alpha_{c}$, and $E_{v}>E_{0}$ as $\alpha>\alpha_{c}$, showing that the variational scheme fails at $\alpha=\alpha_{c}$. Typical results for the variational calculation based on the DOS in the case of $s=0.5$ and $\Delta_{0} / \omega_{D}=0.1$ are shown in Fig. 2. For the Ohmic case with $\Delta_{0} / \omega_{D} \ll 1$, the energy difference $E_{v}-E_{0}$ decreases to zero continuously as $\alpha \rightarrow$ $\alpha_{c}$. The results of the variational calculation based on the DOS is shown in Fig. 3. However, as $\Delta_{0} / \omega_{D}$ increases to some larger values, the self-consistent equation has two non-zero solutions as shown in Fig. 1, and the situation becomes the same as in the sub-Ohmic case.

Analytically, the fail point $\alpha_{c}$ of the variational scheme can be determined by

$$
E_{v}=E_{0} .
$$

In the case of the variational scheme based on the DOS, this leads to

$$
\alpha_{c}=\frac{\Delta_{0}\left(K_{1}-K_{0}\right)}{\int_{0}^{\omega_{D}}\left(\frac{\omega}{\omega_{D}}\right)^{s-1}\left(\frac{K_{1} \Delta_{0}}{\omega+K_{1} \Delta_{0}}\right)^{2} d \omega},
$$

where $K_{0}=\left\langle\phi_{+} \mid \phi_{-}\right\rangle=\exp \left\{-\frac{2}{\pi} \int_{0}^{\omega_{D}} \frac{J(\omega)}{\omega^{2}} d \omega\right\}$, is the adiabatic dressing factor. In the Ohmic case, we have $s=1$ and $K_{0}=0$ and the fail point is

$$
\alpha_{c}=1+K_{1}\left(\Delta_{0} / \omega_{D}\right) .
$$

The above equation is just the crossover point predicted by the variational calculation in the limit of $\Delta_{0} / \omega_{D} \ll$ $1.12,14$ On the other hand, for the variational scheme based on the DSS, the fail point equation tells

$$
\frac{1}{2} \Delta_{0} D=\left.\sum_{k} \sinh ^{2}\left(2 \gamma_{k}\right) \omega_{k}\right|_{\alpha=\alpha_{c}}
$$

where $\gamma_{k}$ is determined by $e^{4 \gamma_{k}}=\sqrt{1+4 D \Delta_{0} c_{k}^{2} / \omega_{k}^{3}}$ and $D=\left\langle\varphi_{+} \mid \varphi_{-}\right\rangle$is the dressing factor $\stackrel{15,16}{16}$ The righthand-side of the above equation represents the energy gain due to the deformation of the phonon state (the squeezed effect) $\stackrel{16}{\underline{16}}$ This energy gain depends on the spectral function as well as the phonon density of states which is assumed to be $\rho(\omega)=\omega^{n-1} / \omega_{D}^{n}$, where $n$ is the dimension degree of the bath in the long-wavelength approximation $\frac{15}{\underline{n}}$ In the Ohmic case $(s=1)$, it can be found that

$$
\alpha_{c} \simeq n+1, \quad \text { for } \quad \Delta_{0} / \omega_{D} \ll 1 .
$$




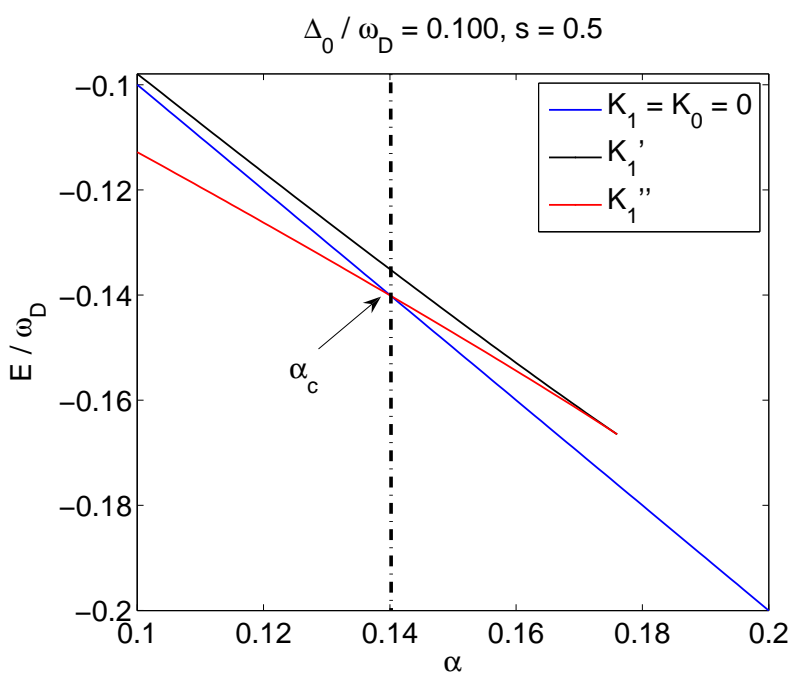

FIG. 2: (Color online) Variation of both the variational ground state energy $E_{1}\left(K_{1}^{\prime \prime}\right)$ and the displaced oscillator ground state energy $E_{0}\left(K_{1}=0\right)$ as a function of $\alpha$ in the sub-Ohmic case with $s=0.5$ and $\Delta_{0} / \omega_{D}=0.1 . \quad E_{1}\left(K_{1}^{\prime}\right)$ represents the energy maximum lies between. For $\alpha>\alpha_{c}$, $E_{1}\left(K_{1}^{\prime \prime}\right)>E_{0}\left(K_{1}=0\right)$ demonstrates the failure of the variational scheme.

Once again this result coincides with the crossover point predicted by the corresponding variational calculation $\stackrel{15,27}{\underline{1}}$ The calculation in the hybrid variational scheme is rather complicated and we find it is hard to find the crossover point analytically in this case.

The above analysis provides a different explanation for the crossover predicted by the variational calculations. When $\alpha<\alpha_{c}$, we have $E_{v}<E_{0}$ and the variational ground state is stable. However, for $\alpha>\alpha_{c}$, the variational scheme fails to give a lower energy and the variational ground state becomes unstable, which results in a transition from the variational ground state to the displaced oscillator ground state at $\alpha=\alpha_{c}$. Since the variational ground state has a non-zero tunneling splitting while the displaced-oscillator ground state has zero tunneling splitting, such a transition is misidentified as the crossover from the delocalized to localized phases, leading to an artifact of phonon-induced localization. It is important to note that the transition point from the variational ground states to the displaced-oscillator ground state is determined by Eq. (14). In the Ohmic case with $\Delta_{0} / \omega_{D} \ll 1$, the transition is continuous, while in the sub-Ohmic case or Ohmic case with a large $\Delta_{0} / \omega_{D}$, the transition is discontinuous and in this case, the transition point is given by Eq. (14) according to Landau theory $\underline{19}$ Based on the above result, it is easy to understand why different variational schemes predicted different crossover points.

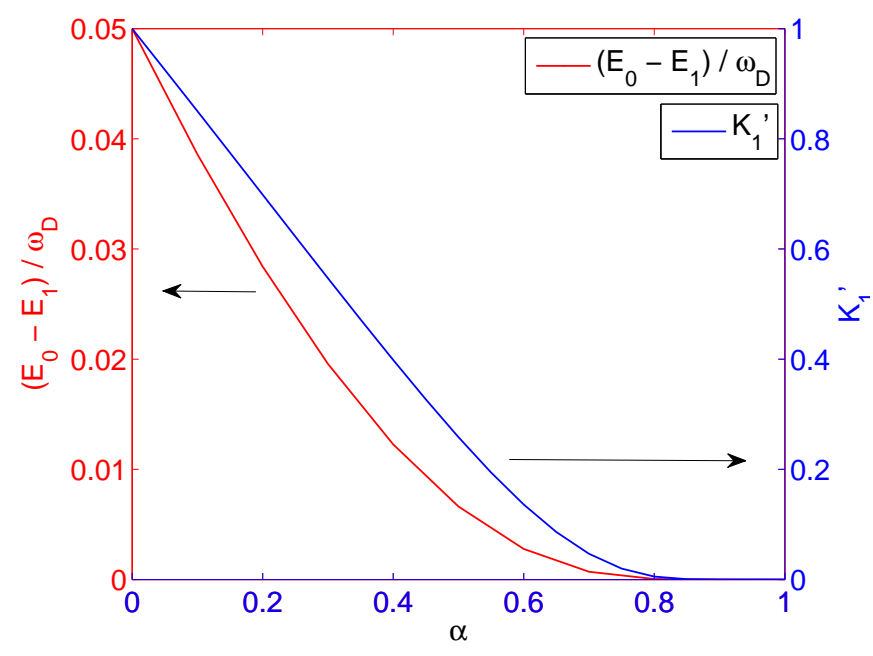

FIG. 3: (Color online) Variation of both the energy difference $\left(E_{0}-E_{1}\right)$ (with scale on the left) and the non-zero solution $K_{1}^{\prime}$ (with scale on the right. Note that this $K_{1}^{\prime}$ is the nonzero solution in the Ohmic case, not one of the solutions in the sub-Ohmic case mentioned above) of self-consistent equation with the dissipation strength $\alpha$ in the Ohmic case with $\Delta_{0} / \omega_{D}=0.1$. As $\alpha$ increases, both $\left(E_{0}-E_{1}\right)$ and $K_{1}^{\prime}$ decrease continuously to zero as $\alpha \rightarrow 1$.

\section{B. Fail point of the variational scheme at $T \neq 0$}

In the case of $T \neq 0$, there is only one kind of variational scheme based on the DOS. The application of other variational schemes mentioned above is in question since the variational scheme based on the DSS was shown to fail in thermodynamical limit $\stackrel{21}{=}$ Variational calculation at $T \neq 0$ is different from the case of $T=0 . \frac{13,18}{1}$ Firstly, we perform a displaced-oscillator transformation to the Hamiltonian given in Eq. (1),

$$
\hat{H}^{\prime}=U^{-1} \hat{H} U=\hat{H}_{0}+\hat{V},
$$

where

$$
U=\exp \left\{\sigma_{z} \sum_{k} f_{k}\left(b_{k}-b_{k}^{\dagger}\right),\right.
$$

and $f_{k}$ is the variational parameter. It can be found that

$$
\begin{gathered}
\hat{H}_{0}=\frac{\Delta}{2} \sigma_{x}+\sum_{k}\left(b_{k}^{\dagger} b_{k}+\frac{1}{2}\right) \omega_{k}+\sigma_{z}^{2} \sum_{k}\left(\omega_{k} f_{k}^{2}-2 f_{k} c_{k}\right), \\
\hat{V}=V_{+} \sigma_{+}+V_{-} \sigma_{-}+V_{0} \sigma_{z},
\end{gathered}
$$

where

$$
V_{+}=V_{-}^{*}=\frac{\Delta_{0}}{2} \exp \left\{-2 \sum_{k} f_{k}\left(b_{k}-b_{k}^{\dagger}\right)\right\}-\frac{\Delta}{2},
$$

$V_{0}=\sum_{k}\left(b_{k}^{\dagger}+b_{k}\right)\left(c_{k}-f_{k}\right)$, and $\Delta$ is the tunneling splitting in the presence of dissipation at $T \neq 0$. Up to the 
second order approximation, the up-bound of the free energy can be found by the Bogoliubov-Feynman theorem and the result is (setting the Boltzmann constant $\left.k_{B}=1\right)^{18}$

$$
A_{B}=-T \ln [2 \cosh (\beta \Delta / 2)]+\sum_{k}\left(\omega_{k} f_{k}^{2}-2 f_{k} c_{k}\right),
$$

where $\beta=1 / T$ and the variational condition in the present case reads

$$
\delta A_{B} / \delta f_{k}=0
$$

which leads to

$$
f_{k}=\frac{c_{k}}{\omega_{k}+\Delta \operatorname{coth}\left(\beta \omega_{k} / 2\right) \tanh (\beta \Delta / 2)},
$$

and a self-consistent equation of the dressing factor $D=$ $\Delta / \Delta_{0}$ can be found

$$
D=\exp \left\{-\alpha \int_{0}^{\omega_{D}} \frac{J(\omega) \operatorname{coth}(\beta \omega / 2) d \omega}{\left[\omega+D \Delta_{0} \operatorname{coth}(\beta \omega / 2) \tanh (\beta \Delta / 2)\right]^{2}}\right\},
$$

substituting the above result back to Eq. (24) and the up-bound of the free energy for the variational thermodynamical ground state can be found. In analog to the case at $T=0$, we have a displaced-oscillator thermodynamical ground state and its up-bound of free energy can be found by setting $f_{k}=c_{k} / \omega_{k}$, the result is

$$
F_{0}=-T \ln 2-\frac{\alpha}{2} \int_{0}^{\omega_{c}} d \omega \frac{J(\omega)}{\omega} .
$$

It is clear that the displaced-oscillator ground state corresponds to the trivial solution of the self-consistent equation (27), e.g., $A_{B}(D=0)=F_{0}$. By the way, as in the case of $T=0$, we should have $A_{B}<F_{0}$ for a successful variational scheme and the fail point equation is

$$
F_{0}=A_{B}
$$

Numerical analysis shows that, for a fixed temperature, the variational scheme fails when $\alpha$ increases to some critical point $\alpha_{c}$, while for a fixed $\alpha$, the variational scheme fails when $T$ increases to some critical point $T_{c}$. Typical results are shown in Fig. 4. As a matter of fact, the situation is more or less that same as in the case of $T=0$. The variational thermodynamical ground state becomes unstable when the fail point is reached and a transition from the variational thermodynamical ground state to the displaced-oscillator thermodynamical ground state happens, which is misidentified as the crossover from the delocalized to localized phases. It is worth noting that such a transition happens for $0<s \leq 2$ in the case of $T \neq 0.22$ Based on the above analysis, the transition for $1<s \leq 2$ (i.e., in the super-Ohmic case) is not a true delocalized-localized phase transition, hence it is not in confliction with the known result.

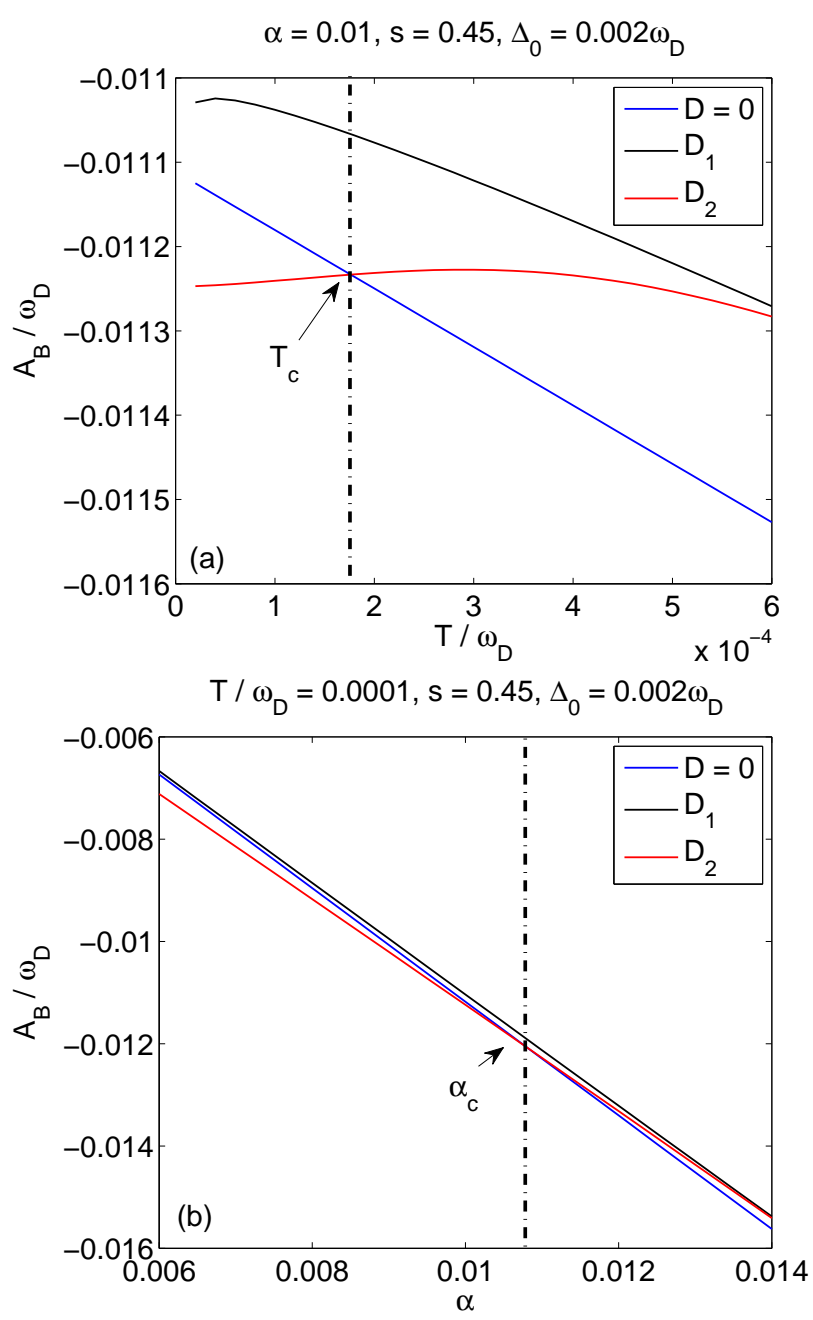

FIG. 4: (Color online) Comparison of the free energies obtained from the self-consistent equation in the case of $s=0.45$ and $\Delta_{0} / \omega_{D}=0.002$. The trivial solution $D=0$ represents the displaced-oscillator thermodynamical ground state. Two non-zero solutions $D_{2}$ and $D_{1}$ represents the variational thermodynamical ground state and a maximum lies between. (a) Illusion for the failure of the variational scheme for a fixed $\alpha=0.01$. At the beginning of the low temperature region, $A_{B}\left(D_{2}\right)<F_{0}=A_{B}(D=0)$ and the variational thermodynamical ground state is stable, when $T$ increases to some critical point $T_{c}$ we have $A_{B}\left(D_{2}\right)=A_{B}(D=0)$. For $T>T_{c}$ we have $A_{B}\left(D_{2}\right)>A_{B}(D=0)$, showing the failure of variational scheme at $T=T_{c}$. (b) Illusion for the failure of the variational scheme for a fixed $T / \omega_{D}=10^{-4}$ in the case of $\Delta_{0} / \omega_{D}=0.002$. The situation is the same as in (a), variational scheme fails when $\alpha>\alpha_{c}$.

\section{FURTHER ANALYSIS ON FAIL POINTS OF THE VARIATIONAL SCHEMES}

In this section, we shall try to answer two questions: why the variational schemes fail and why we have different transition behaviors (continuous and discontinuous) when the fail point is reached? To this goal, we turn to study the oversimplified spin-boson model, i.e., a two- 
level system coupled to just one phonon mode, the Hamiltonian in the single phonon mode case is given by $\underline{4.28}$

$$
\hat{H}_{1}=-\frac{1}{2} \Delta_{0} \hat{\sigma}_{x}+\omega \hat{b}^{\dagger} \hat{b}+\lambda \hat{\sigma}_{z}\left(\hat{b}^{\dagger}+\hat{b}\right) .
$$

The reason why we study this simple model is as follows. Firstly, the fail point analysis presented above can be used to this model to see whether fail point exists in the single mode case. Secondly, the ground state of this model can be known analytically in an approximate way or more exactly by the numerical diagoanlization. $\stackrel{4.28}{=}$ The comparison between the variational ground state with fail point and the true ground state can help to give the answer.

\section{A. Fail point in the single mode case}

In the case of $T=0$, the fail point analysis for multimode case can be directly generalized to the present case. For example, the fail point equation for this model can be directly deduced from the corresponding equations given before. For clarity, we shall use the variational scheme based on the DOS as an example to show the details. From Eq. (15), one can find that the fail point equation in the present case is given by

$$
\frac{1}{2}\left(k_{1}-k_{0}\right) \Delta_{0}=\left(\lambda_{c} / \omega\right)^{2} \frac{k_{1}^{2}\left(\Delta_{0} / \omega\right)}{\left(1+k_{1} \Delta_{0} / \omega\right)^{2}},
$$

where $k_{1}$ is given by the self-consistent equation

$$
k_{1}=\exp \left\{-\frac{2 \lambda^{2}}{\left(\omega+k_{1} \Delta_{0}\right)^{2}}\right\}
$$

and $k_{0}=e^{-2(\lambda / \omega)^{2}}$. The above equation, in general, have three solutions $k_{0} *, k_{1}^{\prime}$, and $k_{1}^{\prime \prime}$ where $k_{0} *<k_{1}^{\prime}<k_{1}^{\prime \prime}$. Numerical analysis shows there exists a frequency threshold $\omega_{c}$, variational schemes fail only for $\omega<\omega_{c}$ at $\lambda=\lambda_{c}$, which is $\omega$-dependent. It turns out that, for $\omega>\omega_{c}$, the variational ground state is always stable, i.e., $E\left(k_{1}\right)<$ $E\left(k_{0}\right)$, and the tunneling splitting $k_{1} \Delta_{0}$ decreases continuously with increasing $\lambda$. However, for $\omega<\omega_{c}$, the tunneling splitting shows a discontinuous jump as $\lambda$ approaching $\lambda_{c}$, at where the variational ground state becomes unstable. Figure 5 shows the variation of both the energy and tunneling splitting around the fail point. In fact, such a discontinuous jump was firstly reported in Ref. 4 and stated it as an artifact of the variational calculation. Our analysis shows clearly this artifact is due to the fail point of the variational scheme. Nevertheless, the discontinuous jump in the single mode case has some important difference from the discontinuous transition in the multi-mode case. First of all, the discontinuous jump does not exactly lead to a crossover from the delocalized to localized phases since both phases have non-zero tunneling splitting. The most important difference is that the transition is not from the variational ground state to the displaced-oscillator ground state, but to a state closed to it. This is because $k_{0}$ is not a solution of the self-consistent equation (32), a situation that is qualitatively different from the multi-mode case where $K_{0}=0$ is the trivial solution of self-consistent equation (12) in both the Ohmic and sub-Ohmic cases. Since the transition determined from the self-consistent equation can only happens between the solutions of this equation, the transition cannot be from the variational ground state to the displaced-oscillator ground state directly, nevertheless the difference becomes very small when $\omega / \Delta_{0} \leq 1 / 5$.

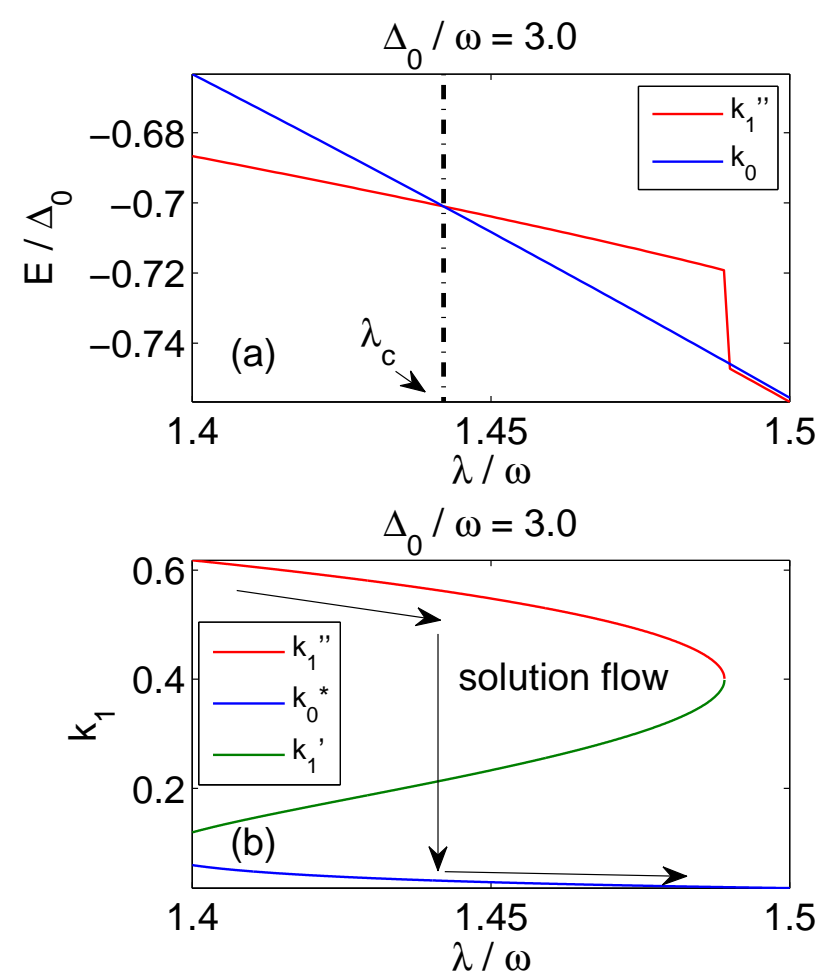

FIG. 5: (Color online) Illusion of the fail point of the variational scheme based on the DOS in the single-mode case with $\Delta_{0} / \omega=3.0$. In the present case, the self-consistent equation has three solutions, $k_{1}^{\prime \prime}, k_{0}^{*}$, and $k_{1}^{\prime}$, which represent respectively the variational ground state, a state closed to displaced-oscillator ground state, and the energy maximum lies between. (a) Variation of the two ground state energies with $\lambda$. The variational ground state energy becomes larger than the displaced-oscillator ground state when $\lambda>\lambda_{c}$, showing the failure of the variational scheme. (b) Solutions of the self-consistent equation as a function of $\lambda$. When the fail point is reached, the variational ground state become unstable and tunneling splitting will have discontinuous jump, i.e., from $k_{1}^{\prime \prime} \Delta_{0}$ to $k_{0}^{*} \Delta_{0}$.

Fail points of other variational schemes can be analyzed in the same way. The situation is almost the same for all three variational schemes except the values of $\omega_{c}$ and the corresponding $\lambda_{c}$. Figure 6 shows the boundary lines of the fail points as a function of $\Delta_{0} / \omega$ and $\lambda / \omega$ for 
all the three variational schemes. As one can see from Fig. 6, all variational schemes fail in the low frequency and strong coupling regions.

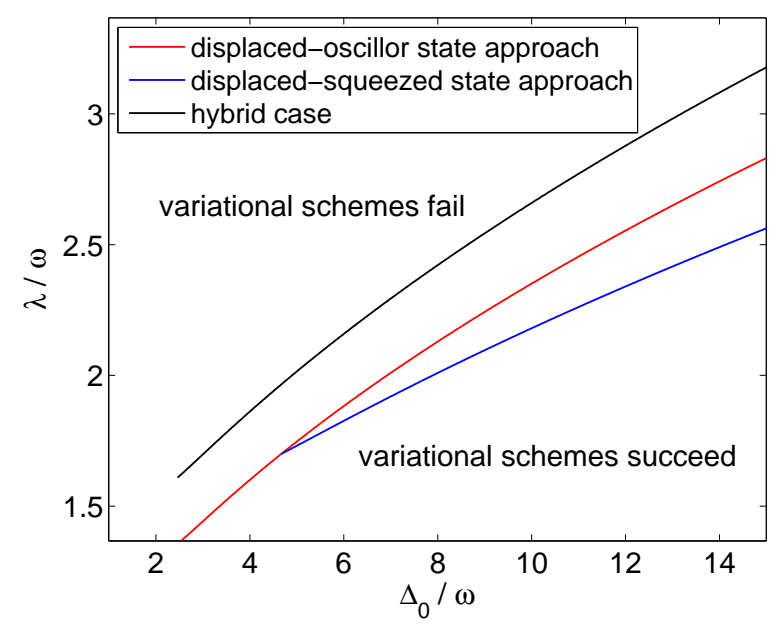

FIG. 6: (Color online) Fail point boundary lines of the three variational schemes in the single-mode case. The starting points of the curves are $\left(\Delta_{0} / \omega_{c} \simeq 2.54 ; \lambda_{c} / \omega \simeq 1.37\right)$, $\left(\Delta_{0} / \omega_{c} \simeq 4.67 ; \lambda_{c} / \omega \simeq 1.70\right)$, and $\left(\Delta_{0} / \omega_{c} \simeq 2.46 ; \lambda_{c} / \omega \simeq\right.$ 1.61) respectively for the DOS, DSS, and hybrid case.

It should be noted that the above analysis cannot be generalized directly to the case of $T \neq 0$. This is because the system with a Hamiltonian given in Eq. (30) is not a true thermodynamical system. To perform the variational calculation as in the multi-mode case, one needs to include the environment as a "thermo-bath", i.e., the effect of the environment is just to keep the single-mode system in thermodynamical equilibrium. Under this sense, we can do a variational calculation based on the DOS and the up-bound of the free energy in the present case is given by

$$
f_{a}=-T \ln \left[2 \cosh \left(\beta k_{2} \Delta_{0} / 2\right)\right]-\omega^{-1}\left(C^{2}-2 C \lambda\right),
$$

where

$$
C=\frac{\lambda}{1+k_{2} \Delta_{0} \omega^{-1} \operatorname{coth}(\beta \omega / 2) \tanh \left(\beta k_{2} \Delta_{0} / 2\right)},
$$

and $k_{2}$ is determined by the self-consistent equation

$$
\ln k_{2}=-\frac{2 \lambda^{2} \operatorname{coth}(\beta \omega / 2)}{\left[\omega+k_{2} \Delta_{0} \operatorname{coth}(\beta \omega / 2) \tanh \left(\beta k_{2} \Delta_{0} / 2\right)\right]^{2}} .
$$

Correspondingly, the up-bound of the free energy of the displaced-oscillator ground state is

$$
f_{0}=-T \ln \left[2 \cosh \left(\beta k_{3} \Delta_{0} / 2\right)\right]-\lambda^{2} / \omega,
$$

where $k_{3}=\exp \left\{-2(\lambda / \omega)^{2} \operatorname{coth}(\beta \omega / 2)\right\}$. Using the above result, one can make analysis on the stability of variational thermodynamical ground state as before. As expected, it turns out that the situation is almost the same as in the case of $T=0$. There is a frequency threshold $\omega_{c}$. For $\omega>\omega_{c}$, we have $f_{a}<f_{0}$, i.e., variational thermodynamical ground state is always stable for all the coupling range and $k_{2} \Delta_{0}$ decreases continuously with increasing $\lambda$; however, for $\omega<\omega_{c}$, variational thermodynamical ground state becomes unstable as the coupling reaches some critical value $\lambda_{c}$ which is again $\omega$-dependent. Tunneling splitting shows a discontinuous jump when the fail point is reached. In the case of $T \neq 0$, the fail point can be reached in another way; that is, for a given coupling $\lambda$, one can find that the variational scheme fails when temperature rises to some critical value $T_{c}$, at where tunneling splitting also shows a discontinuous jump. The boundary line of the fail point for the variational scheme based on the DOS in the case of $T \neq 0$ is shown in Fig. 7. The boundary lowers down as the temperature increases, which implies that the variational scheme fails at a lower $\lambda_{c}$ as $T$ increases.

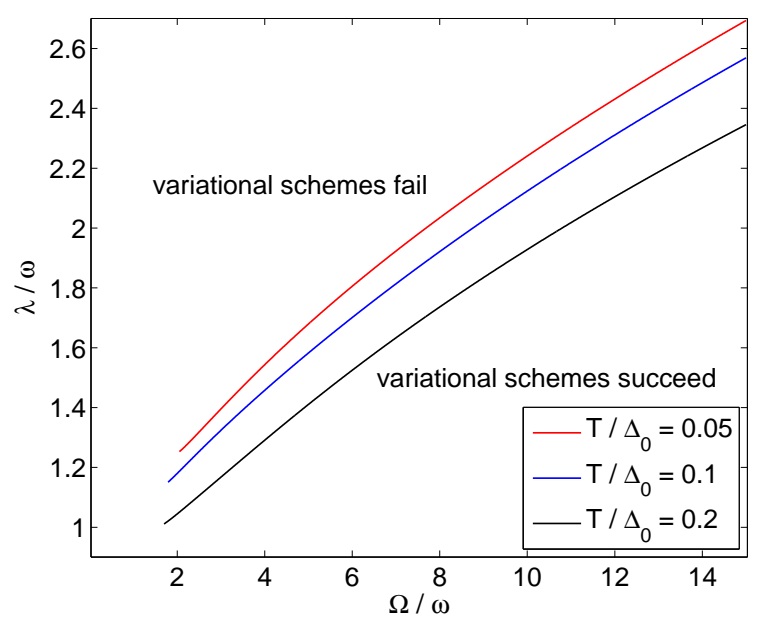

FIG. 7: (Color online) Boundary lines of fail point for variational scheme based on the DOS in the single-mode case at $T \neq 0$. As $T$ raises, the boundary lines lowers down, showing that the variational scheme fails at a lower $\lambda_{c}$ as $T$ increases.

Why variational schemes fail in the low frequency region? Physically, in the high frequency region with $\omega / \Delta_{0} \gg 1$, the first term in Eq. (1) or (30) can be treated as a small perturbation, hence the DOS is a good trial ground state for the variational calculation; however, in the low frequency region with $\Delta_{0} / \omega \gg 1$, the DOS is not appropriate to serve as the trial ground state since the term $\frac{1}{2} \Delta_{0} \hat{\sigma}_{x}$ makes the main contribution in the single mode case. As one can see from the analysis presented in Ref. 4 , in the low frequency region, the true ground state of the phonon bath in the single mode case is a combination of the two DOS with opposite displacement, i.e.,

$$
\left|\phi_{p h}\right\rangle=\frac{1}{\sqrt{1+a^{2}}}\left[e^{-g_{1}\left(\hat{b}^{\dagger}-\hat{b}\right)}+a e^{g_{2}\left(\hat{b}^{\dagger}-\hat{b}\right)}\right]|0\rangle,
$$

where $g_{1,2}$ and $a$ are functions of $\lambda / \Delta_{0}$ and $\omega / \Delta_{0}$. Numerical analysis shows that $\left|\phi_{p h}\right\rangle$ gives a very accurate 
description for the phonon ground state in the low frequency region with strong couplings. Obviously, $\left|\phi_{p h}\right\rangle$ can be described by neither a DOS-like nor a DSS-like state, showing that both the DOS and DSS can not serve as the trial ground states in the low frequency region with strong couplings. As shown in Ref. 4, a variational scheme by taking a trial ground state as $\left|\phi_{p h}\right\rangle$ shows no discontinuous jump. We have made numerical analysis on the energy of the variational ground state based on $\left|\phi_{p h}\right\rangle$, which is found to be always stable against the displacedoscillator ground state in all the coupling regime. This result clearly shows that the failure of the variational scheme is due to the invalid trial ground state in the low frequency regions. The above analysis provides the answer to why the variational schemes based on both the DOS and DSS always fail in low frequency region with strong couplings.

It should be noted that the low frequency modes play important roles in the localized transition. As one can see from the self-consistent equations (12) or (27), the integral can be divided into two parts like, $\int_{0}^{\omega_{D}} \rightarrow$ $\int_{0}^{\omega_{c}}+\int_{\omega_{c}}^{\omega_{D}}$, without the contribution of the low frequency modes lie in $\left(0, \omega_{c}\right)$, there is no localization for any finite $\alpha$. In other words, it is the low frequency modes lead to localization. The failure of the variational schemes in the low frequency regions implies that the variational calculations fail to take account of the contribution of the low frequency modes to the localization, making the predicted localization in question.

\section{B. Continuous or discontinuous crossover}

To the first order approximation (i.e., by omitting the cooperative effect between different phonon modes), the result of multi-mode case can be considered as a combination of all the phonon modes lie in $\left(0, \omega_{D}\right)$ with a frequency-dependent weight. Generally speaking, the parameter $s$ in the spectral function $J(\omega)$ determines the weight, while for counting the contribution of the phonon modes that have fail point; the parameter $\Delta_{0} / \omega_{D}$ is therefore important and the boundary $\omega_{c}$ is in scale of $\Delta_{0}$ as shown in Fig. 6. Let us take the variational scheme based on the DOS as an example. Giving that $\Delta_{0} / \omega_{D}=0.1$, one can see from Fig. 6 that $\omega_{c} / \Delta_{0} \simeq 1 / 3$, which implies that the frequency modes with frequency $0<\omega<0.03 \omega_{D}$ have fail points, i.e., about 3 percent phonon modes have fail points. However, if one takes $\Delta_{0} / \omega_{D}=1$ as shown in Fig. 1, then there are about 30 percent phonon modes have fail points. This result shows that the population of the phonon modes having fail points is directly controlled by the value of $\Delta_{0} / \omega_{D}$. Now we know that only the phonon modes have fail points will contribute a discontinuous jump of the tunneling splitting as the coupling reached the critical point. In the Ohmic case with $\Delta_{0} / \omega_{D} \ll 1$, the discontinuous contribution of the low frequency modes at the fail point is overwhelmed by the continuous contribution from the high frequency modes, leading to a continuous transition; however, as $\Delta_{0} / \omega_{D}$ increases to some larger value (say, 0.5 in the DOS case), the increasing population of phonon modes having fail points leads to an observable discontinuous contribution and the transition behavior turns to discontinuous. In the sub-Ohmic case, the weight of the low frequency modes increases so that the discontinuous contribution from phonon modes having fail points becomes observable even when $\Delta_{0} / \omega_{D} \ll 1$, hence the transition is always discontinuous. However, for the same reason, one can easily predict that the discontinuous transitions will be unapparent when $\Delta_{0} / \omega_{D}$ becomes too small. It should be noted that the discontinuous transition in the sub-Ohmic case is just due to the fail point of the variational schemes, not the true crossover behavior of the model since it is not expected to be seen in the NRG or DMRG calculation. $23,24,25$

The above analysis can be generalized to the case of $T \neq 0$. It has been shown that the transition behavior predicted by the variational calculations at $T \neq 0$ is discontinuous for $0<s<2 \underline{22}$ The key point is to understand why the discontinuous transition behavior is extended to the super-Ohmic case. By comparing the two self-consistent equations (12) for $T=0$ and (27) for $T \neq 0$, at $T \neq 0$, the spectral function is effectively modified as $J(\omega) \operatorname{coth}(\beta \omega / 2)$, i.e., an extra factor $\operatorname{coth}(\beta \omega / 2)$ appears in numerator of the integrand. It is obvious that, $\operatorname{coth}(\beta \omega / 2) \propto 1 / \omega$ as $\omega \rightarrow 0$, which implies that, in the low frequency regions, $J(\omega) \operatorname{coth}(\beta \omega / 2) \propto \omega^{s-1}$. In other words, the extra factor $\operatorname{coth}(\beta \omega / 2)$ seriously increases the weight of the low frequency modes having fail points, resulting in a discontinuous transition behavior even in the super-Ohmic case.

Mathematical analysis on the self-consistent equations derived from the variational condition at both $T=0$ and $T \neq 0$ shows there exists a universal $s_{c}$ for all the variational schemes, the predicted transition is always discontinuous for $0<s<s_{c}$, a continuous transition can only be found when $s=s_{c}$ with $\Delta_{0} / \omega_{D} \ll 1$. It was found that $s_{c}=1$ for $T=0$ and $s_{c}=2$ for $T \neq 0.22$ The above analysis clearly shows that the predicted transition behavior depends on both the weight and the population of the low frequency modes.

\section{CONCLUSION AND DISCUSSION}

In conclusion, we have shown that all the variational schemes used in the spin-boson model have fail points. As the fail point is reached, the variational schemes fail to give a lower energy (or lower free energy at $T \neq 0$ ) than the displaced-oscillator ground state, leading to a transition from the variational ground state to the displacedoscillator ground state, which is always misidentified as the crossover from the delocalized to localized phases in a variational calculation. Our analysis show that the existed variational schemes are not suitable for studying the crossover problem in the spin-boson model. The present 
analysis can help to understand the controversy between different variational schemes and discrepancy with other calculations, like the NRG and DMRG. The analysis on the fail point in the single mode case can help to answer why the variational schemes have fail points and how different transition behaviors were found in different conditions. However, the situation is still far from totally satisfactory. One intriguing fact is that the "fake" crossover point predicted by the variational calculation based on the DOS is found to be in good agreement with the results obtained by other methods including the NRG calculation for both the Ohmic and sub-Ohmic cases $\frac{5,19,23}{\underline{2}}$ In the present stage, it is not yet clear if this is just a coincidence. One possible explanation is that since the population of the low frequency modes having fail points is very small in the case of $\Delta_{0} / \omega_{D} \ll 1$, the contribution of them is small comparing with the rest of the phonon bath. Our DMRG calculation also assures that the variational ground state based on the DOS is a good approximation to the true ground state before the transition happens ${ }^{25}$ Nevertheless, as we have mentioned before, the phonon-induced localization is mainly due to the low frequency modes, combined with the discontinuous transition behavior in the sub-Ohmic case, the answer is not very convincing. Perhaps one can find that answer by developing a variational scheme without fail point and this work is now under process. The present result can also shed some lights on the failure of the variational calculation on the self-trapped transition in ploaron and exciton systems. In fact, the present analysis is readily applicable to the discontinuous transition predicted by the variational calculation gave in Ref. 4 , but further analyses are needed for other cases $\frac{3.11}{.1}$

\section{Acknowledgments}

This work was supported by a grant from the Natural Science Foundation of China under Grant No. 10575045.
* Author to whom correspondence should be addressed: tzhidech@jnu.edu.cn

1 G. Grosso and G. P. Parravicini, Solid State Physics, (Academic Press, NY, 2000).

2 T. D. Lee, F. E. Low, and D. Pines, Phys. Rev. 90, 297 (1953).

3 D. M. Larsen, Phys. Rev. 187, 1147 (1969).

4 H. B. Shore and L. M. Sander, Phys. Rev. B 7, 4537 (1973).

5 A. J. Leggett, S. Chakravarty, A. T. Dorsey, M. P. A. Fisher, A. Garg, and W. Zwerger, Rev. Mod. Phys. 59, 1 (1987) and references there in.

6 U. Weiss, Quantum Dissipative Systems, (World Scientific Singapore 1999).

7 H. Spohn and R. Dümcke, J. Stat. Phys. 41, 389 (1985).

8 S. K. Kehrein and A. Mielke, Phys. Lett. A 219, 313 (1996).

9 S. Sachdev, Quantum Phase Transition, (Cambridge University Press, Cambridge, England, 1999).

10 M. Vojta, Rep. Prog. Phys. 66, 2069 (2003).

11 B. Gerlach and H. Löwen, Rev. Mod. Phys. 63, 63 (1991) and references there in.

12 W. Zwerger, Z. Phys. B 53, 53 (1983).

13 R. Silbey and R. A. Harris, J. Chem. Phys. 80, 2615 (1984).

14 Y. H. Nie, J. J. Liang, Y. L. Shi, and J. Q. Liang, Eur. Phys. J. B 43, 125 (2005).
15 H. Chen and L. Yu, Phys. Rev. B 45, 13753 (1992).

16 H. Chen, Y. M. Zhang, and X. Wu, Phys. Rev. B 39, 546(1989); Phys. Rev. B 40, 11326 (1989).

17 B. Dutta and A. M.Jayannavar, Phys. Rev. B 49, 3604 (1994).

18 A. Chin and M. Turlakov, Phys. Rev. B 73, 075311 (2006).

19 H. Wong and Z. D. Chen, Phys. Rev. B 76, 077301 (2007).

20 W. Chen and Z. D. Chen, Chin. Phys. Lett. 24, 1140 (2007).

21 A. W. Chin, Phys. Rev. B 76, 201307(R) (2007).

22 Z. D. Chen and Z. L. Hou, Chin. Phys. B 17 (7), (accepted).

23 R. Bulla, N.-H. Tong, and M. Vojta, Phys. Rev. Lett. 91, 170601 (2003).

24 R. Bulla, H. J. Lee, N. H. Tong, and M. Vojta, Phys. Rev. B 71, 045122 (2005).

25 H. Wong and Z. D. Chen, Phys. Rev. B 77, 174305 (2008).

26 G. D. Mahan, Many-Particle Physics, (Plenum Press, New York 1990).

27 Using the notation of Ref. 15, the result is $\alpha_{c}=3-2 \lambda$. From Eq. (11) in this reference, it is found that $s=2 \lambda+n-$ 1 , for $s=1$, we have $2 \lambda=2-n$, this leads to $3-2 \lambda=n+1$.

28 E. K. Irish, J. Gea-Banacloche, I. Martin, and K. C. Schwab, Phys. Rev. B 72, 195410 (2005). 